\title{
Ophthalmic Radiotherapy: Plaques and Implants
}

\author{
Paul T. Finger, Mark J. Rivard, Sonal S. Chaugule, \\ P. Mahesh Shanmugam, Svetlana Saakyan, \\ Hatem Krema, Mandeep S. Sagoo, \\ and Wolfgang A. G. Sauerwein
}

Radiation therapy is an indispensable tool used by eye cancer specialists around the world [1,2]. However, ophthalmic radiation therapy can be expensive and typically requires collaboration between an ophthalmic oncologist, radiation oncologist, and a medical physicist. This chapter provides an overview of a basic ophthalmic radiation therapy program as well as indications for irradiation of common ocular malignancies.

Electronic supplementary material The online version of this chapter (https://doi.org/10.1007/978-3-030-18757$\left.6 \_14\right)$ contains supplementary material, which is available to authorized users.

\section{P. T. Finger $(\square)$}

The Eye Cancer Foundation, Inc., The New York Eye Cancer Center, New York University School of Medicine, New York, NY, USA

e-mail: pfinger@eyecancer.com

\section{J. Rivard}

Department of Radiation Oncology, Brown University School of Medicine, Providence, RI, USA e-mail: mark.j.rivard@gmail.com

\section{S. S. Chaugule}

Ophthalmic Plastic Surgery, Orbit and Ocular Oncology, PBMA's H V Desai Eye Hospital, Pune, India

e-mail: schaugule@eyecancercure.com

\section{P. M. Shanmugam}

Vitreoretinal and Oncology Service, Sankara Eye Hospital, Bangalore, India

e-mail: shanmugam1998@yahoo.com
Ionizing radiation has been used to destroy cancers of the eyelids, conjunctiva, iris, retina, choroid, optic nerve, and orbit [3-5]. The most common applications are brachytherapy (implanted and surface applicators), external beam radiation therapy (EBRT), and rarely interstitial orbital brachytherapy techniques. Most of these approaches have a surgical component.

\section{S. Saakyan}

Department of Ocular Oncology, Moscow Helmholtz Research Institute of Eye Diseases, Moscow, Russia e-mail: svsaakyan@yandex.ru

\section{H. Krema}

Department of Ophthalmology and Vision Sciences, Princess Margaret Hospital, Toronto, ON, Canada e-mail: Hatem.krema@unh.ca

\section{S. Sagoo}

Ocular Oncology Service, Moorfields Eye Hospital and Retinoblastoma Service, Royal London Hospital, UCL Institute of Ophthalmology, London, UK e-mail: Mandeep.sagoo@moorfields.nhs.uk

\section{W. A. G. Sauerwein}

Department of Radiation Oncology, Strahlenklinik, University Hospital Essen, Essen, Germany e-mail: w.sauerwein@uni-due.de 


\section{Programmatic Overview}

Herein, we describe technical aspects required to start a radiation therapy program. These include specific imaging protocols for treatment planning of the various lesion types, specifically tumor-imaging or simpler nonimaging methods, quality assurance for the radiation sources encompassing the implanted brachytherapy device, and sterilization methods and radiation surveys of the operating theater and patient before/after implantation and explantation. Once the agreed-upon workflow is established and formalized in writing, it must be approved by the appropriate local radiation control agencies. All participants in each process should have reached a consensus as how to proceed. Any new staff should be trained, and there should also be annual retraining for all participants. It is helpful to have test conditions to evaluate staff comfort and proficiency with the multitude of tasks comprising ocular brachytherapy. An open environment will aid the long-term objective of having a safe and successful ocular brachytherapy program.

\section{Costs}

To be successful, all programs should perform a financial analysis before starting any procedures and then reviewed at least annually to ensure that prior assumptions were accurate and to capture looked-over items. In doing this financial analysis, a proposed workflow should be defined and include the following components: initial patient consultation, image acquisition, treatment planning, source ordering, quality assurance measures, treatment device sterilization, device implantation, radiation surveys, patient discharge (if applicable), device explant, post-explant wound care, device cleaning, and source disposal (if applicable). Estimates for each component cannot be given herein as every center will have unique circumstances that should be carefully assessed in the financial analysis. However, it is known that brachytherapy is both a cost-effective and clinically successful means of treating numerous ocular diseases. With consistent high-quality care and reproducible treatments for a given prescription, there should be a simple means for billing code entry by the staff performing the procedures or their designates.

In general, a brachytherapy program is typically less expensive to start than EBRT. Brachytherapy requires the purchase of implantable materials. For example, ruthenium$106\left({ }^{106} \mathrm{Ru}\right)$ applicators are solid brachytherapy sources, which are relatively inexpensive because they can be reused for multiple temporary implants over their longer half-life (372 days). In contrast, low-energy eye plaques [e.g., palladium-103 $\left({ }^{103} \mathrm{Pd}\right)$, iodine-125 $\left({ }^{125} \mathrm{I}\right)$ ] are relatively more expensive because radioactive seeds must be purchased, inserted in plaque shells, and calculated to the prescribed dose. ${ }^{125} \mathrm{I}$ and ${ }^{103} \mathrm{Pd}$ seeds exhibit half-lives of 59.4 and 17 days, respectively [2]. Therefore, they can be reused but only after reassessment of their current strength. It is important to note that seeded plaques are more versatile compared to solid sources. Seeded plaques are capable of treating larger tumors, can be customized to load the seeds eccentrically to try to spare normal tissues from radiation exposure, seeds can be mixed to modulate intraocular radiation distribution and require smaller margins (2-3 mm) [1, 6-8]. Thus, low-energy seeds can be affixed into custom-designed plaques to treat tumors of the iris, ciliary body, optic disc and conjunctiva more pre-cisely than a solid source plaque. Though most western centers use one plaque for each patient, high volume centers have decreased the cost per patient by reusing low-energy seeds and seed carriers.

In contrast, EBRT (e.g., photon, electron, proton) carries the highest startup cost [9]. In addition to the large capital cost of the linear or particle radiation accelerator, EBRT typically requires large, expensive facilities and radiationshielded rooms. Although brachytherapy and proton EBRT provide comparable tumor control rates, brachytherapy produces the most conformal radiation dose distributions (within the eye) with less long-term radiation toxicity, particularly to anterior ocular and adnexal structures $[6,10-12]$. 


\section{Personnel}

\section{The Team: Medical Physicist, Radiation Oncologist, and Ophthalmic Oncologist}

Radiation therapy personnel are required. The medical physicist (MP) and radiation oncologist (RO) are typically responsible for collaborating on radiation source selection, dose calculation (dose rate, implant duration, and target volume) $[1,2]$. The MP usually constructs radioactive sources prior to implantation and/or administering EBRT. However, it is the ophthalmic oncologist (OO) who selects the patients and monitors for radiation side-effects. Therefore, the $\mathrm{OO}$ should become familiar with both MP and RO aspects of therapy [1]. For the purpose of this chapter, the OO's primary role is to make or confirm the diagnosis, biopsy tumors (when necessary), and surgically insert radioactive devices. In some countries, the $\mathrm{OO}$ can obtain licensing for the selection and prescription of radioactive plaques. Similarly, the RO may assume the role of MP.

\section{Indications for Surgery}

\section{Available Evidence-Based Guidelines}

\section{Uveal Melanoma}

Commonly treated with eye and vision-sparing surgical "plaque" irradiation [13]. In 2014, consensus guidelines were published by an international multicenter Ophthalmic Oncology Task Force (OOTF) that included 47 radiation oncologists, medical physicists, and ophthalmic oncologists from 10 countries. From this work, there was level 1 consensus that all centers:

1. Adopt the use of the American Joint Committee on Cancer (AJCC) or Union for International Cancer Control (UICC) staging systems to facilitate communication.

2. Brachytherapy procedures are performed at specialized medical centers with expertise.

3. These centers were defined as having a subspecialty-trained ophthalmic surgeon, a radiation oncologist, and a medical physicist who are all familiar with the AAPM TG-129 report [2].

\section{Uveal Melanoma Case Selection}

1. A clinical diagnosis of uveal melanoma is adequate (biopsy is not needed).

2. Small melanomas can be treated at the eye cancer specialist's discretion.

3. AJCC-UICC T1, T2, T3, and T4a-d uveal melanoma patients can be treated after counseling about likely vision, eye retention, and local control outcomes.

4. Patients with peripapillary, sub-, and perifoveal melanoma and those with exudative retinal detachments should be counseled about poorer resultant vision and local control outcomes.

5. Tumors with $\mathrm{T} 4 \mathrm{e}$ extraocular extension, diameters that exceed the limits of brachytherapy, blind painful eyes, or no light perception vision are not suitable.

ABS = American Brachytherapy Society; AJCC $=$ American Joint Committee on Cancer, UICC = Union International for Cancer Control; ${ }^{106} \mathrm{Ru}$ and ${ }^{90} \mathrm{Sr}$ plaques are less accommodating for nodular extra-scleral extension. (Reprinted by permission of Brachytherapy [1].)

\section{Retinoblastoma Case Selection}

The most common reasons to use plaque radiation for retinoblastoma is for eye and vision salvage after systemic, regional, or intraocular chemotherapy [1, 14]. An example: residual or relapsed RB that failed local control with chemoreduction and large enough not to be controlled by focal laser and/or cryotherapy. Prospects for sight-threatening complications should be discussed with the family as part of the consent process, through retinoblastoma typically requires a lower tumor apex dose than uveal melanoma. Further, the ABS groups achieved Level 2 consensus that the most common indication for plaque treatment of retinoblastoma is when the tumor is anterior to the equator in unilaterally affected children [1]. Exceptions include when there is evidence of anterior chamber tumor. The ABS-OOTF recommended that children at risk for extraocular RB undergo systemic staging [1]. Episcleral brachytherapy can be used to treat unilateral unifocal tumor or as an adjunct to treat chemoresistant tumors, post-chemotherapy 
residue showing signs of recurrence, and too large to treat with laser or cryotherapy. However, note chemotherapy treated eyes appear more likely to develop intraocular radiation vasculopathy. Lastly, specialized orbital applicators have been devised to irradiate orbital RB recurrence [1].

\section{Orbital Brachytherapy Case Selection}

There are three general indications for orbital brachytherapy implants: extrascleral extension of uveal melanoma, orbital invastion of adnexal tumors and recurrent retinoblastoma $[15,16]$. Extrascleral extension of intraocular cancers presents unique challenges. Small amounts of uveal melanoma extrascleral extension are often treated with local plaque therapy with or without subsequent scleral patch grafting. However, in cases where the eye has been removed and residual tumor (microscopic disease) is suspected, postoperative radiation therapy has been employed to sterilize any remaining orbital cancer. EBRT ( $\approx 40-50$ Gy) with 6 MV photons has been prescribed [4]. However, such treatment typically results in entry dose-associated eye lash and brow loss, dry socket, a sunken orbit, or orbital bone dysgenesis (in children) [4]. There exist alternative high-dose-rate interstitial brachytherapy methods to temporarily implant the radiation within the orbit. These techniques increase the dose within the targeted orbit while decreasing the dose to the eye lids and brow [15]. More cosmetically acceptable, this technique has been reported to yield equivalent local control and improved cosmesis $[4,15,16]$.

\section{Intraocular Tumors}

\section{Uveal Melanoma}

Uveal melanoma is the most primary common ocular tumor treated with implant radiation therapy in the developed world [17]. Methods of radiation plaque construction and dosimetry were reviewed by the American Association of Physicists in Medicine (AAPM) Task Group 129 in 2012 [2]. Similarly, open-access consensus clinical treatment guidelines were published in 2013 by the American Brachytherapy Society (ABS) [1].
Both of these guidelines offered the most widely accepted, evidence-based consensus from teams of eye cancer specialists. Invaluable for a new center, this information includes methods of dosimetry, quality assurance, and case selection $[1,2]$. Though both apply to uveal melanoma, aspects also are relevant for selected intraocular retinoblastomas, as well as other intraocular tumors.

\section{Vascular Tumors of the Retina and Choroid}

Peripapillary or large retinal angiomas can be treated with brachytherapy. Circumscribed or diffuse choroidal hemangiomas smaller than the largest available episcleral brachytherapy implant can also be treated. However, when compared to brachytherapy, low-dose (16-24 Gy), photon-based external beam radiation therapy of choroidal hemangiomas will typically offer relative-sparing vision-critical intraocular structures $[4,18,19,31]$.

\section{Uveal Metastasis}

While most choroidal metastases are treated with nonsurgical EBRT, indications for plaque radiation therapy include radiation resistant tumors (e.g., renal cell, adenoid cystic carcinoma), when prior ocular radiation does not permit treatment of subsequent metastasis and when a patient refuses EBRT [4].

\section{Ocular Adnexal Tumors}

\section{Conjunctival Tumors (Melanoma, Squamous Carcinoma)}

Conjunctival tumors (melanoma, squamous carcinoma) can be treated with radiation. Melanomas are more commonly treated with these methods in that squamous carcinomas are more amenable to surgery with adjuvant cryotherapy and more recently topical chemotherapy [20]. Specialized plaques have been used to treat the ocular surface malignancies in select centers using a variety of radionuclides. Though the use of ${ }^{106} \mathrm{Ru}$ and ${ }^{125} \mathrm{I}$ plaques has been reported, by far the least expensive (but not widely available) method is strontium- $90\left({ }^{90} \mathrm{Sr}\right)$ brachytherapy due to its 28.9-year half-life. 


\section{Orbital Tumors (Melanoma, Retinoblastoma)}

Orbital Tumors (melanoma, retinoblastoma) can also receive curative and/or adjuvant ophthalmic brachytherapy [4]. Albeit rare, most commonly reported are treatments of residual tumors after enucleation of the eye. In addition, orbital adenoid cystic and basal cell carcinomas have been treated with orbital radiation implants. By far the most common use of orbital radiation therapy (EBRT) is for lymphoma (not covered in this chapter).

\section{Presurgical Preparations}

\section{Ocular Imaging}

Intraocular tumor imaging is used to determine whether or not the patient is eligible for brachytherapy treatment and then to dictate how the treatment should proceed [1]. Tumor dimensions are determined by clinical examination, photographic imaging, and ultrasonographic measurements. Typically performed with ultrasound (US) in A-scan or B-scan modes eye cancer specialists determine the largest basal dimension and furthest apical height of intraocular tumors. Other preimplant imaging modalities include optical coherence tomography and fundus autofluorescence imaging which offer more information about tumor extent (within tissues).

Small iris tumors are best localized and measured by slit lamp and gonioscopic photography as well as high-frequency ultrasound imaging. The clinically measured size and extent should be mapped in the chart. In addition, the tumour can be measured with calipers. Immidiately prior to surgery, the tumour borders should be drawn on the cornea and sclera with a marking pen. Transillumination may be used to help define the posterior extent of anterior segment tumours that allow production of an episcleral shadow. The surgeon should place the transilluminator in the quadrant opposite that of the tumor (or through the pupil if better) to produce a tumor shadow to be marked with a marking pen. Transillumination is often difficult in individuals with pigmented fundi and with tumors obscured by the ciliary body band.
In such situations and posteriorly placed tumors, the anterior and lateral margins of the tumor may be localized on to the scleral surface using indirect ophthalmoscopy with scleral depression. Such localization techniques are equivalent to those used for scleral buckling surgery [1].

\section{Treatment Planning}

Tumor measurements and distances to critical intraocular structure drive the resultant radiation treatment plan. Depending on the center, treatment planning can range from a simple calculation of the time to deliver the prescribed radiation dosage to a desired depth, to the sophisticated fusion of multiple radiographic imaging modalities used to measure the lesion, its location and distances to critical intraocular structures. Such computer-based radiographic imaging can be helpful for calculation of 3D dose distributions and the biologic effective dose.

As time marches forward, there have been significant advances in ocular brachytherapy treatment planning. The COMS approach derived dose to points based on characterizing ${ }^{125} \mathrm{I}$ brachytherapy seeds as point sources on a template diagram in a liquid water environment without concern for high-Z materials such as the treatment device or bony anatomy [21]. More recently, image-guided (CT/MRI) brachytherapy treatment planning has permitted more accurate approximations of volumetric dose information throughout the lesion and adjacent healthy structures. At this time, there exists no widely accepted radiographic image guided method or application for intraocular tumors [1].

To our knowledge, there is only one treatment planning system (TPS) created for eye plaque brachytherapy [22]. Many centers employ generic brachytherapy systems altered to perform for ocular use. Commissioning of any treatment planning system will include checks on the linearity of dose with prescription, tests of exponential decay based on the radionuclide half-life, spot checks of the reference dose distribution for a given source or brachytherapy treatment device with that replicated by the TPS, checks 
of volumetric calculations for accurate structure assessments, checks of dose-volume calculations for depiction of accurate 3D volumetric information, and many other basic features as prevalent in brachytherapy TPSs. Further, modern systems may include the ability to depict source collimation or dose differences between tissue and water [23].

\section{Device Preparation}

Most centers purchase solid or assemble seeded treatment devices. Therefore, each center must have a protocol to validate their treatment devices to match what is expected and characterized within the brachytherapy TPS. Commissioning the device will include comparisons of the manufacturer-reported dimensions and compositions to hands-on measurements and also those used in the TPS.

COMS-type plaques and other multicomponent treatment devices consist of a combination of several components that must be compatible. Sometimes the components (such as the glue necessary to hold the device together) are not explicitly approved by local governmental or regulatory agencies for medical purposes such as sterilization and implantation. To be proactive for regulatory audits, the center will need to document the suitability of all components and their intended use in their ocular brachytherapy program.

Some brachytherapy devices such as ${ }^{106} \mathrm{Ru} /{ }^{106} \mathrm{Rh}$ episcleral plaque have a built-in radiation source and are intended to be used on multiple patients. Other devices may have a nonradioactive plaque into which low-energy photon-emitting brachytherapy seeds (i.e., ${ }^{103} \mathrm{Pd}$, ${ }^{125} \mathrm{I}$, or ${ }^{131} \mathrm{Cs}$ ) are loaded. These seeds may be reused depending on the source half-life, rate of patient accrual, and variety of prescriptions. In both cases, care must be taken to inspect (preceding first use and subsequent reuse) to ensure radioactive capsule integrity. Evaluation (mathematical or physical) of source strength is generally made prior to each use. Measurements of source strength require a re-entrant well-type air ionization chamber and seed holder with calibration for a given source model that is traceable to a primary standards calibration laboratory. In the United States of America, this is achieved through calibrating the chamber and seed holder at one of three Accredited Dosimetry Calibration Laboratories, which are traceable to the National Institute of Standards and Technology (NIST) [24]. Centers located elsewhere should follow local regulations. While not technically challenging, sterilization of the ocular brachytherapy device requires diverse attributes and coordination across multiple disciplines (Video 14.1).

Multicomponent devices will have differing or unspecified sterilization procedures with a limited number of permissible sterilization cycles (pertinent for devices to be used on multiple patients). Given the sensitive nature of some components such as glue or a seed holder, care must be taken to establish a sterilization program that accommodates limitations of all components yet provides timely and effective sterilization $[1,2]$.

Whichever approaches are taken, it is recommended that a patient database be established from the inception of an ocular brachytherapy program to capture information on various patient specific aspects of the procedure. This database can include imaging datasets and treatment planning information in addition to simple fields like implant/explant dates and times; names of participating staff; lesion height and basal dimensions; prescription dose, extent, and radionuclide; and dose-volume information for adjacent healthy structures. These data may be stored within the electronic health record system for later query.

\section{Therapy Techniques}

\section{Orbital Brachytherapy for Residual Orbital Melanoma}

We recommend that this procedure be performed 4-6 weeks after enucleation or other subtotal excisional surgery to allow wound healing [15]. When used for orbital extrascleral melanoma 
extension, the temporary prosthesis or conformer is removed and the patient placed under general anesthesia. The eyelids and conjunctival surfaces are prepared with povidone-iodine antiseptic (Table 14.1). The orbit is inspected for the condition of the conjunctival wound and the position of the orbital implant. A marking pen is used to define evenly spaced $(1 \mathrm{~cm})$ sites for catheter placement. The sites should be located just within the bony rim of the orbital bone by palpation. Care is taken to avoid critical structures (e.g., lacrimal drainage system, superior and inferior orbital nerves, and anterior ethmoid artery). Using the marks as a guide, transcutaneous \#11 blade incisions should be performed (sequentially) as to create 3-mm wide orbitotomy sites. The aforementioned brachytherapy catheters were marked with ink to indicate when they are $<4.5 \mathrm{~cm}$ deep into the orbit. A brachytherapy button is placed onto the catheter to aid cutaneous fixation.

Then, a bimanual catheter implantation technique is used to guide the trochar toward the orbital apex. While the catheter enters the orbit, the surgeon uses on finger to palpate the catheter tip as it traverses through the orbital soft tissues. Once in place, with the catheter tip at the orbital apex, a cutaneous 4- 0 suture is placed though the brachytherapy button to anchor it in place. Then, the catheters trocar is withdrawn. This series of steps is repeated with each catheter until there are $8-10$ evenly spaced catheters in place. Then medical grade glue is placed to affix the trocars to the brachytherapy buttons and allowed to dry (Video 14.2).

Prior to being discharged to the recovery room, the catheters are trimmed to $5 \mathrm{~cm}$ in length and covered with a protective gauze dressing. Upon recovery from anesthesia, the orbit is imaged by computed tomography for treatment planning by the medical physicist.

\section{Orbital Brachytherapy for Extrascleral Uveal Melanoma}

High-dose-rate (HDR) brachytherapy is performed in a shielded room using a remote after
Table 14.1 Ophthalmic radiosurgery equipment and devices

\begin{tabular}{l}
\hline Surgical equipment/materials \\
\hline Anesthesia \\
\hline $\begin{array}{l}\text { Sterilization fluid for the plaque or Autoclave for solid } \\
\text { plaques }\end{array}$ \\
\hline Indirect ophthalmoscope \\
\hline 20 diopter lens \\
\hline Scleral depressor \\
\hline Ocular muscle suture material \\
\hline Conjunctival suture material \\
\hline Transilluminator and cable \\
\hline Sterile Q-tips \\
\hline Schepens Retractors \\
\hline Scissors, forceps, speculum \\
\hline Orbital brachytherapy materials \\
\hline Anesthesia \\
\hline \#11 blade and Adsen forceps \\
\hline $8-10$ brachytherapy catheters with trochars \\
\hline $8-10$ brachytherapy buttons \\
\hline 4-0 silk sutures \\
\hline Surg
\end{tabular}

Surgical marking pen

Medical grade epoxy or cyanoacrylate

Surgical dressing

Radiation isolation room

Computed axial tomography scans

HDR Iridium-192 system/treatment planning software/ applicator(s)/shielded room

Select commercial vendors

Gold plaque shells

http://www.trachseldentalstudio.com

Bebig ruthenium plaques

http://www.bebig.com/home/products/ophthalmic_

brachytherapy/ru_106_eye_applicators

Tantalum clips

http://www.novoprecision.com/products/

tantalum-markers

Preloaded plaques

https://www.isoaid.com/prod_pages/eye-plaque

Survey meters

https://www.environmental-expert.com/companies/

keyword-geiger-counter-27616

Radioactive seeds

Best Medical

http://www.teambest.com

BEBIG

http://

www.bebig.com/home/products/seed_brachytherapy

IsoAid

https://www.isoaid.com

Theragenics

https://www.theragenics.com

BXTAccelyon

https://www.bxt-accelyon.com

Resotech

http://www.resotech.ru 
loader that houses an ${ }^{192} \mathrm{Ir}$ source. Under mild sedation and with pain medication, the orbital catheters are attached to the mechanical remote after-loader which sends the source down the catheters into the orbit for the prescribed dwell time, and are then retracted. Nine to ten treatment fractions are typically delivered (BID) over 5 consecutive days. At the end of treatment, $3-5 \mathrm{~cm}^{3}$ of lidocaine is placed into the orbit 15 min prior to extraction of the catheters. Once removed, the $3 \mathrm{~mm}$ cutaneous wounds are treated with topical antibiotic ointment and the ocular prosthesis is replaced.

\section{Orbital Brachy-Boost Technique}

In an effort to treat microscopic residual radiation-resistant tumor (e.g., adenoid cystic carcinoma, hemangiopericytoma, invasive carcinomas) HDR radiation can be applied to the tumor bed [4]. While the surgery is similar to the aforementioned technique (for treatment of melanomatous orbit extension), a lower brachytherapy dose is employed in addition to a subsequent overlay of EBRT. The result intensifies treatment of the tumor bed with relative sparing of the rest of the orbit. This eye and vision sparing HDR orbital brachytherapy technique is typically employed as an alternative to orbital exenteration.

\section{Anesthesia for Radioactive Plaque Surgery}

General anesthesia is preferred. This is because introduction of $3-5 \mathrm{~cm}^{3}$ of orbital fluid can limit the surgeons view and increase the difficulty of plaque placement. However, these procedures can be performed with local assisted surgery if needed, particularly for iris, ciliary body and anterior uveal tumors.

In that episcleral brachytherapy can be performed under peribulbar or retrobulbar anesthesia, we recommend using a mixture of $2 \%$ xylocaine and $0.5 \%$ bupivacaine. Caution to be taken to avoid inadvertent globe perforation which can increase the risk of orbital tumor dissemination. Vitreoretinal management of complications of globe perforation is both complicated and controversial.

\section{Surgical Steps for Radioactive Plaque Surgery}

The chart should be reviewed immediately prior to implantation surgery. Eye lid skin and periorbital area is sterilized with $10 \%$ povidone iodine solution with application of $5 \%$ povidone iodine eye drops in the conjunctival cul de sac. Then the patient should be draped with only the operative eye exposed for surgery. Then a secondary examination (ophthalmoscopy or ultrasound imaging) should be performed to confirm the presence of the tumor and condition of the eye. An eyelid speculum is placed. Then a limbal conjunctival periotomy is followed by opening of the intermuscular septa (Tenon's fascia) in all four quadrants. This is performed using a curved tenotomy scissors which is placed with closed blades in the episcleral space between the muscles. The scissors are opened in the orbit and withdrawn with open blades thereby lysing the intermuscular septa.

\section{Anterior Plaques}

Melanomas of the anterior uvea, ciliary body, and iris are commonly treated with plaque radiation [1, 17] (Video 14.3). Such treatment offers a method to cure the patient without intraocular surgery and often preserves the iris and its function. However, epicorneal plaque position can be painful and cause corneal erosions. This is why centers place a thin layer of donor tissue (e.g., amniotic membrane) as a buffer between the plaque and the cornea $[25,26]$. In addition, insertion is complicated by "backward" plaque placement where the plaque eyelets are rotated onto the sclera as to avoid eyelet corneal suturing (see youtube.com/watch?v=B8IxecQCGOQ). 


\section{Circumpapillary Plaques}

While the intraocular optic disc diameter is typically $1.8 \mathrm{~mm}$, behind the eye the optic nerve is encased within the optic nerve sheath (ONS). The retrobulbar optic nerve sheath diameter is typically 5-6 mm, which means that a plaque placed against the ONS can only reach to $1.5 \mathrm{~mm}$ from the optic disc [3, 27-29]. The ONS presents a significant obstruction to normal radioactive plaque placement, defined by the ABS as covering the tumor plus a $2-3 \mathrm{~mm}$ margin. In order for a plaque to overcome the ONS diameter, 8-mm wide (variable depth) slots have been cut from COMStype plaques. In that the seeds are the radioactive sources, the space within the slot can be filled with radiation. However, this requires more complex medical physics planning that guides placement of seeds within the plaque [27]. Due to their sharp penumbra and the $8-\mathrm{mm}$ slot distance, complete tumor coverage or "normal plaque placement" cannot be achieved with solid ${ }^{106} \mathrm{Ru}$ plaques.

\section{Radioactive Plaque Placement}

Most uveal melanomas are located in the posterior choroid. Herein we include a movie showing plaque insertion for a posterior choroidal melanoma (Video 14.4). However, melanomas can occur beneath a variety of extraocular muscles as well as near the optic nerve. Any muscles that might interfere with plaque placement or displace the plaque during treatment should be temporarily relocated to a location that will not disturb the plaque. This typically requires standard disinsertion on locking sutures. They will be returned to their original insertions at the time of plaque removal. Permanent diplopia is uncommon [30].

Transillumination of the eye (transocular or transpupillary) will allow the surgeon to view the tumors shadow. The edges of the shadow are marked as well as the $2-3 \mathrm{~mm}$ surrounding free margin with tissue dye on the sclera. Scleral depression, diathermy marking, and/or focal light transillumination may be required for patients with darkly pigmented choroid.
A dummy plaque is placed on the sclera overlying the tumor and is anchored to the sclera using sutures passed through the eyelet of the plaque. Temporary knots are used to secure the dummy plaque. Intraoperative ultrasound (with the probe wrapped in a sterile sleeve) should be performed to ensure accurate localization of the plaque. Once ensured, the dummy plaque is removed and the radioactive plaque is secured in place using the aforementioned sutures.

In some centers, the radioactive plaque is used instead of a dummy plaque, thus avoiding one step. This change increases irradiation of the surgeon, eliminates possible differences in dummy-active plaque suture-related placement, allows for ultrasonographic verification of the actual radioactive device and decreases surgical time. After plaque placement, the conjunctiva is closed with absorbable sutures. Peribulbar infusion and topical antibiotic and steroid are placed. The eye is covered with a soft pad and a lead shield. Removal of the plaque can also be performed under local or general anesthesia. When the plaque has been placed to treat conjunctival lesions, it can be removed under topical anesthesia.

\section{The Russian Experience with Strontium-90}

\section{Case Selection}

Conjunctival melanoma, squamous carcinoma, and lymphomas may be treated. Brachytherapy of biopsy proven tumors with the exception of uveal melanoma. Tumor thickness should not exceed 3-4 mm. Maximum diameter is practically irrelevant, because the irradiation can be performed in several fields. Margins extend 3-4 mm beyond the visible tumor (on every side). In cases of thicker conjunctival tumors, surgical removal is performed prior to brachytherapy.

Contraindications:

1. Full lid-thickness infiltration.

2. Intraocular and orbital extension. 
Methods:

1. Brachytherapy performed in a reclining chair.

2. Local anesthesia.

3. Head fixation.

4. Strontium-90 plaque (Resotech) is applied to the targeted zone, using a rod (plaque holder) fixed to a headlamp retainer.

5. Single dose applied over 10-30 min.

Dose (cumulative dose at depth):

1. For melanoma, 200 Gy; carcinoma, 120150 Gy; lymphoma, 90-110 Gy.

2. Single dose in a depth, 10-15 Gy.

Strontium-90 for uveal melanoma:

1. Tumor thickness up to $3.5 \mathrm{~mm}$.

2. Dose to the apex 200-230 Gy.

Strontium-90 for retinoblastoma:

1. Tumor thickness up to $3.5 \mathrm{~mm}$.

2. Dose to the apex, 120-150 Gy.

\section{Postsurgical Procedures}

The patient is treated with topical steroid antibiotic eye drops on a tapering schedule for 4 weeks and is examined periodically depending on the type of tumor and plaque treatment.

\section{Radiation Surveys and Regulations}

After each case (insertion and removal), the patient's eye and operating room are assessed to ensure that emitted radiation falls within the prescribed and acceptable range. Precautions and safety measures are detailed to the patient and attenders. Please consult your governmental regulatory authority governing the use of radiation. Each locality typically prescribes the practices related to patient handling after brachytherapy. For instance, in India patients are hospitalized for the duration of treatment. In
New York City, using ${ }^{125} \mathrm{I}$ and ${ }^{103} \mathrm{Pd}$, they are sent home or to a hotel until plaque removal.

Governing regulatory agencies for most centers require radiation surveys to ensure a radiologically safe environment for the staff, patient, and general public. You should consult your government regulatory agency to determine require radiation safety procedure. The practice of radiation surveys should follow the radiation source(s) towards documenting the environment before and after their introduction.

Specifically, this may include receipt of the sources from the manufacturer with wipe tests and radiation survey of the shipping package; wipe tests and radiation survey of the brachytherapy source(s) upon opening the shipping package; radiation survey of the assembled brachytherapy treatment device preceding sterilization; radiation survey of the operating theater and the patient preceding implant; radiation survey of the operating theater, patient, and sterilization container following implant; radiation survey of the operating theater, patient, and sterilization container preceding explant; and radiation survey of the operating theater, patient, and sterilization container following explant. Expected and maximum permissible exposure rates should be included in a standardized form. Survey instrumentation should be calibrated for the radiation quality and expected exposure rates.

\section{Summary}

The authors of this chapter recognize the significant challenges that face any center starting a new ophthalmic radiation therapy program. However, those who are willing to organize this effort will likely see generations of patients benefitting from sight and life-saving treatment.

This chapter was created to serve as a starting point for ophthalmologists, radiation oncologists, and medical physicists around the world. Herein, we pledge to share our knowledge in support to your efforts. 


\section{References}

1. American Brachytherapy Society - Ophthalmic Oncology Task Force. The American Brachytherapy Society consensus guidelines for plaque brachytherapy of uveal melanoma and retinoblastoma. Brachytherapy. 2014;13(1):1-14.

2. Chiu-Tsao ST, Astrahan MA, Finger PT, et al. Dosimetry of ${ }^{125} \mathrm{I}$ and ${ }^{103} \mathrm{Pd}$ COMS eye plaques for intraocular tumors: report of task group 129 by the AAPM and ABS. Med Phys. 2012;39(10):6161-84.

3. Finger PT. Radiation therapy for choroidal melanoma. Surv Ophthalmol. 1997;42(3):215-32.

4. Finger PT. Radiation therapy for orbital tumors: concepts, current use, and ophthalmic radiation side effects. Surv Ophthalmol. 2009;54(5):545-68.

5. Saakyan SV, Amiryan AG, Valskiy VV, Mironova IS. Plaque radiotherapy for anterior uveal melanomas. Vestn Oftalmol. 2015;131(2):5-12.

6. Rivard MJ, Melhus CS, Sioshansi S, Morr J. The impact of prescription depth, dose rate, plaque size, and source loading on the central axis using ${ }^{103} \mathrm{Pd},{ }^{125} \mathrm{I}$, and ${ }^{131} \mathrm{Cs}$. Brachytherapy. 2008;7(4):327-35.

7. Fluhs D, Anastassiou G, Wening J, Sauerwein W, Bornfeld N. The design and the dosimetry of binuclide radioactive ophthalmic applicators. Med Phys. 2004;31(6):1481-8.

8. Gagne NL, Rivard MJ. Quantifying the dosimetric influences of radiation coverage and brachytherapy implant placement uncertainty on eye plaque size selection. Brachytherapy. 2013;12(5):508-20.

9. None. Proton therapy appears to be less cost-effective. Cancer Discov. 2013;3(2):OF2.

10. Krema H, Heydarian M, Beiki-Ardakani A, et al. A comparison between ${ }^{125}$ Iodine brachytherapy and stereotactic radiotherapy in the management of juxtapapillary choroidal melanoma. Br J Ophthalmol. 2013;97(3):327-32.

11. Yousef YA, Finger PT. Lack of radiation maculopathy after palladium-103 plaque radiotherapy for iris melanoma. Int J Radiat Oncol Biol Phys. 2012;83(4):1107-12.

12. Wilson MW, Hungerford JL. Comparison of episcleral plaque and proton beam radiation therapy for the treatment of choroidal melanoma. Ophthalmology. 1999;106(8):1579-87.

13. Kivela T, Simpson ER, Grossniklaus HE, Jager MJ, Singh AD, Caminal JM, Pavlick, et al. Uveal melanoma. In: Amin M, Edge SB, Greene FL, et al., editors. The American Joint Committee on cancer staging manual. 8th ed. New York: Springer; 2017. p. 805-18.

14. Mallipatna AC, Gallie BL, Chevez-Barrios P, Lumbroso-Le Rouic L, Chantada GL, Doz F, Brisse $\mathrm{HJ}$, et al. Retinoblastoma. In: Amin MB, Edge S, Greene F, Byrd DR, Brookland RK, Washington MK, et al., editors. The AJCC cancer staging manual. 8th ed. New York: Springer; 2017. p. 819-31.

15. Finger PT, Tena LB, Semenova E, Aridgides P, Choi WH. Extrascleral extension of choroidal melanoma: post-enucleation high-dose-rate inter- stitial brachytherapy of the orbit. Brachytherapy. 2014;13(3):275-80.

16. Stannard C, Maree G, Munro R, Lecuona K, Sauerwein W. Iodine-125 orbital brachytherapy with a prosthetic implant in situ. Strahlenther Onkol. 2011;187(5):322-7.

17. Finger PT. Intraocular melanoma. In: DeVita JVT, Lawrence TS, Rosenberg SA, editors. Cancer: principles and practice of oncology, vol. 1. 10th ed. Philadelphia: Wolter Kluwer, Lippincott, Williams and Wilkins; 2014. p. 1770-9.

18. Madreperla SA. Choroidal hemangioma treated with photodynamic therapy using verteporfin. Arch Ophthalmol. 2001;119(11):1606-10.

19. Aizman A, Finger PT, Shabto U, Szechter A, Berson A. Palladium $103\left({ }^{103} \mathrm{Pd}\right)$ plaque radiation therapy for circumscribed choroidal hemangioma with retinal detachment. Arch Ophthalmol. 2004;122(11):1652-6.

20. Pe'er J. Ocular surface squamous neoplasia: evidence for topical chemotherapy. Int Ophthalmol Clin. 2015;55(1):9-21.

21. Collaborative Ocular Melanoma Study Group. Ch 12: radiation therapy. In: Service NTI, editor. COMS manual of procedures PB95-179693. Springfield: National Technical Information Service; 1995.

22. Astrahan MA. Improved treatment planning for COMS eye plaques. Int J Radiat Oncol Biol Phys. 2005;61(4):1227-42.

23. Rivard MJ, Chiu-Tsao S-T, Finger PT, et al. Comparison of dose calculation methods for brachytherapy of intraocular tumors. Med Phys. 2011;38(1):306-16.

24. DeWerd LA, Huq MS, Das IJ, et al. Procedures for establishing and maintaining consistent air-kerma strength standards for low-energy, photon-emitting brachytherapy sources: recommendations of the Calibration Laboratory Accreditation Subcommittee of the American Association of Physicists in Medicine. Med Phys. 2004;31(3):675-81.

25 . Finger PT. Finger's amniotic membrane buffer technique: protecting the cornea during radiation plaque therapy. Arch Ophthalmol. 2008;126(4):531-4.

26. Semenova E, Finger PT. Amniotic membrane corneal buffering during plaque radiation therapy for anterior uveal melanoma. Ophthalmic Surg Lasers Imaging Retina. 2013;44(5):477-82.

27. Finger PT, Chin KJ, Tena LB. A five-year study of slotted eye plaque radiation therapy for choroidal melanoma: near, touching, or surrounding the optic nerve. Ophthalmology. 2012;119(2):415-22.

28. Sagoo MS, Shields CL, Emrich J, Mashayekhi A, Komarnicky L, Shields JA. Plaque radiotherapy for juxtapapillary choroidal melanoma: treatment complications and visual outcomes in 650 consecutive cases. JAMA Ophthalmol. 2014;132(6):697-702.

29. Sagoo MS, Shields CL, Mashayekhi A, et al. Plaque radiotherapy for choroidal melanoma encircling the optic disc (circumpapillary choroidal melanoma). Arch Ophthalmol. 2007;125(9):1202-9.

30. Nagendran ST, Finger PT, Campolattaro BN. Extraocular muscle repositioning and diplo- 
pia: associated with ophthalmic plaque radiation therapy for choroidal melanoma. Ophthalmology. 2014;121(11):2268-74.

31. PaulT Finger, Radiation therapy for exudative choroidal hemangioma. Indian Journal of Ophthalmology 2019;67(5):579.

\section{Suggested Reading}

http://www.ijo.in/article.asp?issn=0301-4738;year=2019 ; volume $=67 ;$ issue $=5 ;$ spage $=579 ;$ epage $=581 ;$ aulast $=\mathrm{F}$ inger

Open Access This chapter is licensed under the terms of the Creative Commons Attribution 4.0 International License (http://creativecommons.org/licenses/by/4.0/), which permits use, sharing, adaptation, distribution and reproduction in any medium or format, as long as you give appropriate credit to the original author(s) and the source, provide a link to the Creative Commons license and indicate if changes were made.

The images or other third party material in this chapter are included in the chapter's Creative Commons license, unless indicated otherwise in a credit line to the material. If material is not included in the chapter's Creative Commons license and your intended use is not permitted by statutory regulation or exceeds the permitted use, you will need to obtain permission directly from the copyright holder. 